\title{
Lectura Dantis: Purgatorio XI
}

\author{
MICHAEL SCHWARZE (Konstanz)
}

\begin{abstract}
Riassunto: Un'analisi di Purgatorio XI, il canto della superbia, mette in luce come nella Commedia i peccati possano conservare anche degli aspetti positivi. I tre superbi incontrati da Dante (Omberto Aldobrandeschi, Oderisi da Gubbio e Provenzan Salvani), benché penitenti, non mostrano un atteggiamento totalmente improntato a quella humilitas che sarebbe lecito attendersi da chi si trova nel Purgatorio. All'interno del canto, »ingegno« e »disio de l'eccellenza« non rappresentano soltanto le cause principali della futile brama di gloria che caratterizza gli animi superbi, ma sembrano assumere anche una funzione positiva. $\grave{E}$ dunque possibile individuare un itinerarium ad Deum alternativo rispetto a quello professato dal Padre Nostro ad apertura del canto: un itinerario che non passa per la »via diritta« (Inf. I, 3), bensì prevede un »altro vïaggio« (Inf. I, 9), una via terrena che non esclude l'uso dell'»ingegno« e il »disio de l'eccellenza«. Così Dante ci consegna sottobanco una inaudita rivalutazione delle capacità umane, che si rivelano non puramente negative - cause della miseria hominis -, bensì, parzialmente e velatamente, basi di una dignitas hominis.
\end{abstract}

In Anbetracht des Rahmenthemas der diesjährigen Jahrestagung der Deutschen Dante-Gesellschaft mag es einigermaßen überraschen, dass hier für die Lectura Dantis ein Gesang des Purgatorio gewählt worden ist. Denn, so mag man sich fragen, worin liegt der Gewinn, wenn man sich der »Anthropologie der Hölle« gewissermaßen von der Höhe des Läuterungsberges aus nähert? Welcher Zusammenhang besteht zwischen Purgatorio XI, dem Gesang über den Hochmut, und der Frage, ob die menschlichen Verfehlungen außer in ihrer negativen Ausprägung als Sünde in der Commedia auch eine grundlegend positive Wertigkeit besitzen?

Um diesen Fragen nachzugehen, habe ich besagten elften Gesang der zweiten Cantica ausgewählt. ${ }^{1}$ Zwei Gründe haben mich dazu bewogen:

\footnotetext{
${ }^{1}$ Auch zu Purgatorio XI hat die Dantephilologie eine solche Vielzahl an Auslegungen und Kommentaren hervorgebracht, dass ein selektiver Umgang mit der Forschungsliteratur unumgänglich ist. So zählt etwa die partielle Bibliographie der Società Dantesca Italiana, die kommentierte Ausgaben nicht berücksichtigt, für den Zeitraum seit 1972 für den Gesang 94 Titel (www. danteonli-
} 
Der eine ist, dass die Seelen auf dem ersten Rundsims des Berges mit der superbia ein Laster sühnen, das im theologischen Denken des 13. und 14. Jahrhunderts als Ursprung aller Sünden galt. Im Zentrum dieses Gesangs steht damit ein vitium, das allen konkreten Vergehen, die im Inferno von den Verdammten auf ewig gebüßt werden, als »initium omnis peccati« (Sir 10, 15) zugrunde liegt. ${ }^{2}$ Dies mag auch der Grund dafür sein, dass Dante für den Hochmut nicht eigens einen Höllenkreis vorgesehen hat - das Laster zählt gewissermaßen zur Grundausstattung der Menschen. Dementsprechend groß ist die Zahl derjenigen, die Dante in der Hölle ausdrücklich als hochmütig bezeichnet. ${ }^{3}$

Die Wahl dieses Canto beruht zweitens darauf, dass die Ethik des Purgatorio systematisch betrachtet dem irdischen Leben deutlich näher steht als die beiden an ihn grenzenden Jenseitsreiche. Der Unterschied liegt wesentlich darin, dass dem Läuterungsberg, verstanden als jenseitigem Bewährungsraum der büßenden Seelen, eine zeitliche Dimension zu Eigen ist, die dem menschlichen Zeitverständnis sehr nahe kommt. Denn es herrscht hier eine Vorstellung von Zeit vor, die im Kontrast zur temporalen Statik von Inferno und Paradiso die Kategorie der Entwicklung kennt. Diese konkretisiert sich eben in der Läuterung der Seelen und beschreibt idealiter einen Lernprozess, in dem die Schuld des Einzelnen abgetragen wird. Erreicht ist dieses Ziel in der Commedia, wie Wehle gezeigt hat, wenn Dante auf dem Gipfel des Berges im paradiso terrestre »die Läuterung des Purgatoriums auch an sich selbst vollzogen« hat und nicht zuletzt erkennt, »worin denn Sinn und Wert des

ne.it, [27.3.2011]). Im Folgenden wird daher vornehmlich auf Studien Bezug genommen, die von unmittelbarer Relevanz für die hier vertretene Argumentation sind. Letztere folgt im Wesentlichen dem methodischen Ansatz, den Text der Commedia - selbstverständlich unter voller Respektierung seiner philologischen Gegebenheiten - konsequent im Hinblick darauf zu prüfen, wie kohärent Dante in den exemplarischen Episoden mit den ethischen Konsequenzen verfährt, die sein moraltheologisches System impliziert.

${ }^{2}$ Siehe ganz ähnlich Moralia XXXI 45 des von Dante intensiv studierten Gregor des Großen, der von der »vitiorum regina superbia« spricht. Die Bedeutung des Intertexts aus dem Buch Jesus Sirach hat jüngst G. Polimeni hervorgehoben: »Purgatorio X-XI-XII«, in: Benedetta Quadrio (Hrsg.), Esperimenti danteschi. Purgatorio 2009, Genova 2010, S. 105-133, hier S. 106-108. Der superbia kam im traditionellen Sündenregister seit Gregor dem Großen der Primat in der Genealogie des Bösen zu.

${ }^{3}$ Siehe zum Hochmut in der Commedia konzise und mit entsprechenden Belegen den Eintrag »Superbia« von Fiorenzo Forti in der Enciclopedia dantesca, S. 484-487. 
Menschen für sich selbst genommen besteht, unterhalb seiner göttlichen Letztbestimmung. $\ll^{4}$

Es deutet sich in dem Gesagten bereits an, dass die Reinigung sich der Anlage des Purgatorio folgend in doppelter Weise vollzieht: ${ }^{5} \mathrm{Ge}-$ sühnt wird das jeweilige Vergehen zum einen symbolisch durch das Erleiden einer körperlichen Qual. In unserem Gesang besteht es darin, dass die Hochmütigen die Last von Steinen ertragen müssen, was sie gleichsam zu kriechenden Würmern macht. ${ }^{6}$ Vom Gewicht gebeugt, den Blick zu Boden bezwungen und betend gehen sie auf unterschiedliche Weise gepeinigt »zur Reinigung vom trüben Dunst der Welt « ${ }^{7}$ mühselig die erste cornice hinan:

quell'ombre orando, andavan sotto 'l pondo,

simile a quel che talvolta si sogna,

disparmente angosciate tutte a tondo

e lasse su per la prima cornice,

purgando la caligine del mondo. (Purg. XI, 26-30)

Neben dem physischen Leiden beinhaltet die Läuterung zum anderen einen kognitiven Prozess, der darin besteht, dass die zur Sünde neigende Vernunft des Einzelnen eine Korrektur erfährt und auf den Pfad der Tugendhaftigkeit zurückkehrt. In diesem Übergang wird der eigene Irrtum

${ }^{4}$ Winfried Wehle, ») Rückkehr nach Eden<. Über Dantes Wissenschaft vom Glück in der Commedia«, in: Deutsches Dante-Jahrbuch 78 (2003), S. 13-66, Zitate S. 24.

${ }^{5}$ Siehe zur grundlegenden »Zweiteilung des Läuterungsprozesses« Andreas Kablitz, »Jenseitige Kunst oder Gott als Bildhauer«, in: ders./Gerhard Neumann (Hrsg.), Mimesis und Simulation, Freiburg i.Br. 1998, S. 309-356, hier S. 320323.

${ }^{6}$ Le Opere di Dante Alighieri, edizione nazionale, VII: La Commedia secondo l'antica vulgata, a cura di Giorgio Petrocchi, Milano 1966-1967, hier: S. 3: Purgatorio, X, 115 f. Zitiert wird die Commedia im Folgenden im Fließtext unter Verwendung der Sigle »Purg.« und Angabe der Verse.

${ }^{7}$ Alle deutschen Übersetzungen aus der Commedia folgen, sofern nicht ausdrücklich markiert, dem zweiten Band der exzellenten Prosa-Übersetzung von Hartmut Köhler, der mir seine Fassung von Purg. XI liebenswürdigerweise bereits vorab zur Verfügung gestellt hatte. Dante Alighieri, La Commedia - Die Göttliche Komödie II. Purgatorio/Läuterungsberg, Italienisch/Deutsch, in Prosa übersetzt und kommentiert von Hartmut Köhler, Stuttgart 2011, hier S. 209. Zitiert wird diese Übersetzung im Folgenden im Fließtext unter Verwendung der Sigle »Köhler« und Angabe der Seitenzahl. 
durch die Wiederherstellung der Einsichtsfähigkeit bereinigt. ${ }^{8}$ Einzig der Läuterungsberg sieht in der Commedia damit die Möglichkeit vor, mittels und mit der Zeit die Verfehlungen wieder gutzumachen, derer sich die Menschen während der Zeit auf Erden schuldig gemacht haben. Prägnant kommt diese Implementierung der menschlichen Zeit in das Purgatorio an einer Stelle in Purg. XXIII zum Ausdruck, wo Dante Forese Donati gegenüber vom Vorpurgatorium als dem Raum spricht, »wo Zeit sich durch Zeit erneuert« - »dove tempo per tempo si ristora« (Purg. XXIII, 84).

Dantes thomistisch geprägtes Interesse an diesem programmatischen Läuterungsgeschehen bestand nach allem, was wir wissen, darin, eine homogenisierende Verbindung zwischen der nichtigen Welt und dem verlässlichen Reich Gottes herzustellen. Dieses Ziel vor Augen verwandelt seine Ethik die physische Erniedrigung in ein Instrument der Erkenntnis. Mit dem Entwurf eines sich mit der Zeit vollziehenden Wandels von der sündhaften Verstrickung hin zum Heil der Erkenntnis Gottes aber bildet der Purgatoriumsberg systematisch zugleich die Entwicklung ab, die der Pilger Dante auf seinem Weg durch das Jenseits erfährt. Insofern kommt die Cantica nicht nur grundsätzlich einem menschlichen Zeitverständnis besonders nahe, sondern betrifft in ihrer moraltheologischen Anlage im Kern und ganz konkret den »Kampf mit dem Weg«, von dem der Dichter in der Commedia rückblickend erzählt. ${ }^{9}$ Auch deshalb scheint mir das Purgatorium in der hier gewählten Perspektive prädisponiert zu sein, um Grundzüge einer Anthropologie des Heils in der Commedia zu eruieren. In besonderem Maße gilt dies für den elften Gesang des Purgatorio.

I.

Wie das Purgatorium gewissermaßen nel mezzo del cammin der Commedia angesiedelt ist, so bezeichnet auch der ausgewählte Canto eine Mitte: Denn er wird in der Art eines Triptychons von zwei Gesängen

\footnotetext{
${ }^{8}$ Erinnert sei in diesem Zusammenhang lediglich an $>$ Vergils $\$ berühmte Charakterisierung der Sünder in Inf. III, 16-18: »Noi siam venuti al loco ov' i' t'ho detto / che tu vedrai le genti dolorose / c'hanno perduto il ben de l'intelletto.« (Kursivierung M.S.).

${ }^{9}$ Im Rahmen des erweiterten Prologs erklärt der Erzähler in Inf. II, 3-6: »e io sol uno / m'apparecchiava a sostener la guerra / sí del cammino e sí de la pietate, / che ritrarrà la mente che non erra.« (Kursivierung M.S.).
} 
umrahmt, deren moraltheologisches Thema ebenfalls die superbia darstellt. Im Mittelpunkt des vorangehenden zehnten Gesangs stehen wunderschöne Hochreliefs, die dem Jenseitswanderer eindrucksvoll Beispiele vorbildhafter Demut vor Augen halten. Das Gegenbild dazu liefert der zwölfte Canto, in dem der viator tief bewegt dreizehn Beispiele bestraften Hochmuts bewundert, die in den Boden eingemeißelt sind. $\mathrm{Zu} \mathrm{Be}-$ rühmtheit sind diese beiden Gesänge dadurch gelangt, dass Dante die Kunstwerke in ihnen als das Produkt einer wahrhaft göttlichen Kunst vorstellt, die Gott faktisch als Bildhauer erscheinen lässt. Diese gewagte Konstruktion impliziert eine Reihe theologischer und kunsttheoretischer Probleme, die in den vergangenen Jahren intensiv diskutiert worden sind. ${ }^{10}$ Sie müssen uns hier nicht primär interessieren, zumal unser $s u$ perbia-Gesang inhaltlich in deutlichem Kontrast zu den beiden ihn begrenzenden steht: Während nämlich in jenen entfernte biblische und mythische Exempla in der Art einer gelehrten Galerie evoziert werden, begegnet Dante im elften Gesang konkret drei Persönlichkeiten der toskanischen Zeitgeschichte, deren Leben vom Hochmut gezeichnet waren. Das in Purgatorio XI dargestellte Geschehen vollzieht damit in drei Episoden jene für die Commedia typische zeit-räumliche Annäherung des Jenseits an den lebendigen poeta pellegrino. Auf diese Weise wird das theoretisch begründete Spannungsverhältnis zwischen irdischem Hochmut und jenseitiger Demut, um das es in den drei Canti geht, in kommunikatives Handeln überführt. Und der soeben skizzierte, normative Prozess der purgatio wird in den Gesprächen, die Dante mit den sich läuternden Seelen führt, gleichsam in einer Folge von drei Einzelfällen vermenschlicht.

Aus der Konfrontation Dantes mit drei historischen Beispielen hochmütiger Lebensführung ergeben sich für den Canto drei Sinnabschnitte. Ihnen ist unvermittelt ein einleitender Abschnitt vorangestellt, in dem der Chor der büßenden superbi das Vater Unser betet. ${ }^{11}$ Im vor-

\footnotetext{
${ }^{10}$ Siehe in dieser Perspektive Kablitz, »Jenseitige Kunst« (wie Anm. 5); Paolo Marini, " >La gloria de la lingua nel trittico dei superbi. Considerazioni sul nodo arte-onore-superbia-umiltà nella 〉Commedia««, in: Italianistica 36 (2007), S. 65-88; Michelangelo Picone, »Dante nel girone dei superbi (Purg. X-XII)«, in: L'Alighieri 46 (2005); Giuseppe Polimeni, »Purgatorio X-XI-XII» (wie Anm. 2); Karlheinz Stierle, »Das System der schönen Künste im >Purgatorio von Dantes Commedia«, in: ders., Ästhetische Rationalität. Kunstwerk und Werkbegriff, München 1997, S. 389-416.

${ }^{11}$ Kollektive Bitt- und Bußgebete werden im Purgatorio wiederholt evoziert: So etwa in Purg. XXIII, 11, wo die Schlemmer weinend den Psalm Labïa mëa,
} 
liegenden, von der Kritik wenig beachteten Fall allerdings sticht die ungewöhnliche Länge von 24 Versen ins Auge. Dazu kommt es, weil Dante die ehemals Hochmütigen gewissermaßen ein amplifiziertes Pater Noster anstimmen lässt, in dem den einzelnen Anrufungen Gottes jeweils ein erklärender Kommentar hinzugefügt ist. ${ }^{12}$ So entspricht zum Beispiel das einleitende $\gg \mathrm{O}$ padre nostro, che ne' cieli stai« (Purg. XI, 1) dem lateinischen »Pater Noster qui es in caelis« $(\mathrm{Mt}, 6,9)$ beinahe wortgleich. Darauf jedoch folgt nicht das zu erwartende "sanctificetur nomen tuum«, welches erst in Vers 4 als »laudato sia 'l tuo nome« paraphrasiert wird, ${ }^{13}$ sondern zwei Verse, die den Inhalt des ersten präzisierend erläutern. Die erste Terzine lautet:

O padre nostro, che ne' cieli stai, non circunscritto, ma per piú amore ch'ai primi effetti di là sú tu hai [...] (Purg. XI, 1-3) ${ }^{14}$

Die Kritik versteht den Kommentar einhellig als Ausdruck bußfertiger Demut der superbi, die mittels des Verweises auf die größere Liebe Gottes zu den unbefleckten Engeln allererst die eigene moralische Defizienz anprangern. ${ }^{15}$ Was der in solcher Weise erweiterte Gebetsanfang jedoch

Domine singen, und in Purg. XXV, 121, wo Dante den Hymnus Summae Deus clementiae vernimmt.

${ }^{12}$ In einem kurzen Artikel, der sich mit den theologischen Implikationen von Purg. XI, 1-3 auseinandersetzt, hat Luigi Peirone das Dantesche Gebet kürzlich als "una sorta di parafrasi del Padre nostro« bezeichnet, das »esplicitamente« die Funktion einer »chiosa chiarificatrice del contenuto" habe. Luigi Peirone, »Il Padre nostro nel Purgatorio dantesco«, in: Tenzone. Revista de la Asociación Complutense de Dantología, Madrid 2008, S. 211-215, hier S. 212. Kablitz, »Jenseitige Kunst« (wie Anm. 5) charakterisiert das Chorgebet treffend als »ein >aufgeklärtes` Vater Unser« (S. 337).

${ }^{13}$ Der Vers modelliert offensichtlich den berühmten Cantico des Franz von Assisi. Siehe zur These eines franziskanischen Subtexts für den gesamten Gesang jüngst Andrea Mazzucchi, »Filigrane francescane tra i superbi. Lettura di Purgatorio XI«, in: Rivista di studi danteschi 8 (2008), S. 42-82; zum Pater Noster insbesondere S. 46-56.

${ }^{14}$ In Köhlers Übersetzung lautet die erste Terzine des Gesangs: »O unser Vater, der du im Himmel bist, nicht um dich von uns abzugrenzen, sondern weil du am meisten liebst, was du dort als erstes gewirkt hast«. Köhler (wie Anm. 7), S. 205 (Kursivierung M.S.)

${ }^{15}$ Chiavacci Leonardi kommentiert: »Non noi, miseri uomini, essi sembrano dire, siamo i più grandi nell'universo, come ci siamo creduti. Da ogni versetto 
im selben Zug indirekt vermittelt, ist eine latente Fragwürdigkeit der Aussagen des Vater Unser - eine Fragwürdigkeit freilich, die Dantes Erweiterungen erst eigens evozieren, um sie im selben Moment zu verneinen. Denn der Hinweis darauf, dass Gott nicht im Himmel ist, um sich von den fehlbaren Menschen abzugrenzen, wirkt ganz so, als ob es nicht selbstverständlich sei, dass seine Allgegenwart auch auf Erden gegeben sei. Dem »padre nostro, che ne' cieli stai«, so lässt die folgende Explikation schließen, scheint hier eine latente Ungewissheit innezuwohnen, die einer ausdrücklichen Begründung bedarf. Und insgesamt gewinnt man den Eindruck, als ob die purgatio in dem Gesang zunächst darin liege, das Gebet des Herrn ausdrücklich vom Zweifel der Menschen zu reinigen.

In der dritten Terzine evoziert das solchermaßen purgierte Vater Unser dabei implizit den unerhörten Gedanken, in das Reich Gottes könnten die Menschen vielleicht nicht nur durch das absolute Geschenk des Allmächtigen gelangen, sondern auch durch den Einsatz »all unserer Geisteskraft«: »Vegna ver' noi la pace del tuo regno / ché noi ad essa non potem da noi, / s'ella non vien, con tutto nostro ingegno « (Purg. XI, 7-9, Kursivierung M.S.). ${ }^{16}$ Das Gebet der bußfertigen Hochmütigen erwähnt die Möglichkeit, die Gnade Gottes lasse sich durch menschliche Anstrengung verdienen, auch hier, um eben diesen Gedanken im selben Zuge mit Nachdruck auszuschließen. Gerade die kategorische Negation des Verdienstgedankens aber rückt ein solchermaßen blasphemisches Gedankenkonzept überhaupt erst in den Horizont des Denkbaren. Diese Lesart wird dadurch bestätigt, dass die Gedankenfigur gleich darauf in ähnlicher Form flüchtig noch einmal wiederkehrt; und zwar in der Bitte, der Allmächtige möge den Sündigen das Böse auf Erden vergeben, ohne dabei »auf unser Verdienst « zu sehen: »E come noi lo mal ch'avem sofferto / perdoniamo a ciascuno, e tu perdona / benigno, e non guardar lo nostro merto.« (Purg. XI, 16-18, Kursivierung M.S.) Die Erwähnung

[...] Dante coglie, estrae un simile spunto di umile meditazione.« (Dante Alighieri, Commedia, con il Commento di Anna Maria Chiavacci Leonardi, vol. II: Purgatorio, Milano ${ }^{5} 2003$, S. 323.) Und Peirone (»Il Padre nostro«, wie Anm. 12) paraphrasiert Dantes vielleicht an Thomas von Aquin geschulte Interpretation des Gebetsanfangs wie folgt: »noi diciamo >che sei nei cieli< perché in quello spazio occupato dai cieli risiedono gli angeli, puri spiriti, che ti amano più di noi.« (S. 214)

${ }^{16}$ Köhler (wie Anm. 7), S. 207 übersetzt: »Der Friede deines Reiches komme zu uns, denn mit all unserer Geisteskraft können wir, wenn er nicht kommt, nicht zu ihm gelangen.« 
des »merto« bringt hier erneut latent die dem Vater Unser fremde Eventualität ins Spiel, die Gnade des Herrn könne sich der Einzelne qua Leistung verdienen. Der Gedanke, der Gütige könne den Menschen aufgrund ihres Tuns auf Erden vergeben, dient damit erneut rhetorisch zur Betonung des Gegenteils. Die Kommentare, mit denen Dante das Pater noster des elften Gesangs versehen hat, haben insofern vorderhand ausgesprochen salvierenden Charakter.

Doch scheint mir, dass sich hinter diesem amplifizierten Vater Unser mehr verbirgt. Im Lichte der folgenden Behandlung der superbia nämlich erweisen sich die latenten Zweifel am Wort des Herrn nicht bloß als rhetorische Strategie, sondern als substantiell äußerst brisant. Dies wird schon in der Wahl der Vertreter der Hoffart ersichtlich, auf die Dante sein fiktives Ich treffen lässt: Es handelt sich erstens um Omberto Aldobrandeschi, der einem alten, »arroganten « Adelsgeschlecht entstammt; zweitens um den zeitgenössischen Miniaturmaler Oderisi, der sich zu Lebzeiten ungebremster künstlerischer Ruhmsucht schuldig gemacht hat; und drittens um den mächtigen Sienesischen Politiker Provenzan Salvani, der für die Anmaßung unumschränkter Machtfülle auf der ersten cornice des Läuterungsberges Buße tut. ${ }^{17}$ Dante hat demnach unter den superbi zwei politisch Mächtige und einen Künstler ausgewählt. Dies aber hat er kaum zufällig getan, sondern zweifelsohne, weil diese beiden >Berufsgruppen ihrem Selbstverständnis entsprechend in der Gefahr stehen, genuin göttliche Eigenschaften anzustreben: Im Falle des Mächtigen liegt die potentielle superbia in der Versuchung vollkommener Allmacht, im Falle des Künstlers darin, dass er sich vollkommene Schöpfungsgewalt attestiert. Die Persönlichkeiten, mit deren Geschichten sich Dante in dem Gesang konfrontiert sieht, stehen demnach bereits strukturell betrachtet in Konkurrenz zu den Fähigkeiten des Allmächtigen, den das Vater Unser anbetet. ${ }^{18}$ Die potentielle Konkurrenz beruht dabei auf Seiten des Machtmenschen wie des Künstlers $a$

\footnotetext{
${ }^{17}$ Die jüngere Kritik betont unter Bezug auf den Ottimo commento, den vermutlich ein Zeitgenosse Dantes verfasste, dass die drei Seelen damit gezielt drei unterschiedliche Ausprägungen der superbia repräsentieren: Umberto die Arroganz, Oderisi die Ruhmsucht und Provenzan die Überheblichkeit. Siehe dazu Mazzucchi (wie Anm. 13), S. 44 und Polimeni (wie Anm. 2), mit dem Fokus auf der vanagloria Oderisis, S. 123-125. Chiavacci Leonardi (wie Anm. 15, S. 316-320) dagegen versteht die drei Männer vornehmlich als historische Figurationen, die »tre tipi di superbia, propri dell'ambiente e del tempo in cui viveva Dante« (S. 316) verkörpern.

${ }^{18}$ Auf diesen Punkt hat bereits Kablitz (wie Anm. 5, S. 318 f.) hingewiesen.
} 
priori auf dem Anspruch, ihre ausgeprägten Fähigkeiten so zum Einsatz zu bringen, dass ihnen außerordentlicher Erfolg zuteil wird. Es sind demnach genau der im Kontext der göttlichen Gnade höchst prekäre Einsatz des »ingegno« und ein explizites Verdienstdenken, welche die Menschen im Rahmen ihrer weltlichen Ordnung in gefährliche Nähe zu ihrem Schöpfergott rücken. Im Folgenden wird zu zeigen sein, wie diese zunächst nur grundsätzlich festzustellende Konkurrenz im Verlauf des elften Gesangs dem Anschein zuwider an Relevanz gewinnt - mit eklatanten Folgen für die Heilsanthropologie der gesamten Commedia.

Wenden wir uns dazu zunächst dem moraltheologischen Programm zu, in dessen Zeichen die drei Männer stehen, auf die Dante trifft. Sie eint der Kontrast zwischen ihrem irdischem Leben, das sich auf einen angeborenen Hochmut zu reduzieren scheint, und dem jenseitigen, in dessen Verlauf sie sich bemühen, das Laster in Demut zu verwandeln. Da es sich bei den Figuren in ihrer Zeit um Prominente handelte, äußerte sich die superbia vor allem in ihrem ungezügelten Streben nach Ehre und Ruhm. Dem entspricht, dass sie nun im Purgatorium, erdrückt von der Last der Steine, Einsicht in die Vergänglichkeit dieser eitlen Ziele erlangen sollen, um den wahren Ruhm Gottes zu erkennen. Das Verständnis von Hochmut, das Dante in diesem Kontext aufruft, stimmt mit der Begriffsbestimmung Thomas von Aquins überein. Dieser begreift die superbia als eine Form egoistischer Selbsterhebung, deren Ziel die Überflügelung des anderen ist. Er beschreibt sie als »amor propriae excellentiae, in quantum ex amore causatur inordinata praesumptio alios superandi. $«{ }^{19}$ In genau diesem Sinne macht der reuige Oderisi $» l o$ gran disio / de l'eccellenza ove mio core intese « (Purg. XI, 86 f.) für den eigenen Hochmut verantwortlich. Den semantischen Aspekt der Erniedrigung der Konkurrenten unterstreicht darüber hinaus wenig später die Belehrung, die Vergil seinem Schüler in Purgatorio XVII zuteil werden lässt. Periphrastisch kennzeichnet er dort den Hochmütigen als einen, der hofft hervorzuragen, damit sein Nächster unterdrückt wird, und der nur deswegen danach strebt, damit der andere von seiner Größe herabgestürzt werden möge:

\footnotetext{
${ }^{19}$ Summa theologica II,2, 162,6. Der Aquinat rekurriert dabei auf Augustinus, der den Hochmut in De Civitate Dei XIV, 13 wie folgt bestimmt: »Quid est autem superbia nisi perversae celsitudinis appetitus?« Einen knappen Überblick über die Bestimmungen des Hochmuts bei Thomas v. Aquin bietet Patrick Boyde, Human vices and human worth in Dante's Comedy, Cambridge 2000, S. $187 \mathrm{f}$.
} 
È chi, per esser suo vicin soppresso, spera eccellenza, e sol per questo brama ch'el sia di sua grandezza in basso messo; (Purg. XVII, 115-117)

Gemäß der theologischen Annahme, sie sei der Ursprung aller menschlichen Laster, erhebt wiederum Oderisi diese superbia schließlich zum Signum der gefallenen Menschheit insgesamt. Der Wille hervorzuragen gerät so zum Synonym für den »eitlen Ruhm allen menschlichen Könnens«. »Oh vana gloria de l'umane posse! « (Purg. XI, 91) ermahnt der bußfertige Oderisi den Menschen Dante emphatisch. ${ }^{20}$ Die Verurteilung des menschlichen Könnens, all seiner Vermögen und Fähigkeiten aber schließt die Qualitäten, die den Machtmenschen zum Machtmenschen und den Künstler zum Künstler machen, unweigerlich ein. Die in dem Canto insistent vorgebrachte Ächtung der superbia weitet sich auf diese Weise zu einer allgemeinen Weltverachtung, zum contemptus mundi aus.

In seiner langen Mahnrede, die in diesem Zusammenhang von zentraler Bedeutung ist, setzt Oderisi diese Verachtung, an den Dichter Dante gerichtet, mittels einer ausdrucksstarken Topik nachdrücklich ins Bild. Das Augenmerk des miniatore gilt dabei der Kurzlebigkeit allen Künstlerruhmes, der wie der Wind ständig die Richtung seinen Namen wechsle:

Non è il mondan romore altro ch'un fiato

di vento, ch'or vien quinci e or vien quindi,

e muta nome perché muta lato. (Purg. XI, 100-102)

Und was wird schon, so fragt Oderisi Dante sinngemäß weiter, von deinem Ruf in tausend Jahren bleiben, geschweige denn angesichts der zeitlichen Dimension des ewigen Lebens? ${ }^{21}$ Vor allem aber vergleicht Oderisi die Vergeblichkeit der vana gloria mit dem Grün auf den Wipfeln, das doch nur allzu kurz halte: »com' poco verde in su la cima dura«! (Purg. XI, 92) Der Einsatz dieser Naturmetaphorik, die an das Welken der Blätter im Herbst gemahnt, ruft insgeheim zugleich den »amato alloro« (Par. I, 15), den geliebten Lorbeer auf, von dem der Au-

\footnotetext{
${ }^{20}$ Die Oderisi in den Mund gelegte Formulierung nimmt unmittelbar Thomas' Wendung von der »inanis vel vana gloria« (Summa theologica II, 2, 132), verstanden als vitium capitale, auf.

${ }^{21}$ Siehe Purg. XI, 103-108.
} 
tor der Commedia als Krönung seines Werkes träumt. ${ }^{22}$ In der Perspektive des sich läuternden Oderisi kann es einen solchen immer grünenden Dichterlohn nicht geben. Als Beleg hierfür führt er drei Beispiele an: Zunächst (Purg. XI, 82-84) nennt er sich selbst, da seine von Dante gepriesenen Illuminierungen »jetzt « gegenüber den Blättern eines gewissen Franco aus Bologna an Ehre eingebüßt hätten. ${ }^{23}$ Sodann (Purg. XI, 94-96) erwähnt er den Maler Cimabue, der glaubte der Beste zu sein, inzwischen aber bereits im Ansehen von Giotto übertroffen werde. Und ebenso sei es den beiden Dichtern namens Guido ergangen, womit, wie Picone gezeigt hat, vermutlich Guittone d'Arezzo und Guido Guinizelli gemeint sind. ${ }^{24}$ Der eine habe dem anderen die "gloria de la lingua« (Purg. XI, 97) ${ }^{25}$ geraubt, vielleicht aber sei schon ein Dritter geboren, »der sie alle beide aus dem Nest stößt.«

Cosí ha tolto l'uno a l'altro Guido

la gloria de la lingua; e forse è nato

chi l'uno e l'altro caccerà del nido. (Purg. XI, 97-99)

${ }^{22}$ Siehe zu Purg. XI, 92 und der Frage, worauf sich die »cima« bezieht, eingehend Robert Hollander, »Dante's Self Laureation (Purgatorio XI, 92)«, in: Rassegna europea di letteratura italiana 3 (1994), S. 35-48, insb. S. 44-48. Den vanitas-Gedanken unterstreichend resümiert er: »Dante does not so much arrogate unto himself the laureation he was never to receive as he is commenting upon the way in which any gift bestowed by fame is transitory and, eventually, unimportant.« (S. 46)

${ }^{23}$ Picone, »Dante nel girone dei superbi« (wie Anm. 10, S. 102) hat in diesem Kontext angemerkt, Dante thematisiere hier implizit ein Beispiel der translatio studii französischer Kunst nach Italien - ein Verdienst, das wiederum erklären könnte, warum hier neben den wesentlich berühmteren Künstlerkollegen Oderisi auftreten darf. Siehe dazu ausführlicher: Giovanni Fallani, »Oderisi da Gubbio e Franco bolognese, ricerche e ipotesi sui codici miniati«, in: ders. (Hrsg.), Dante e la cultura figurativa medievale, Bergamo ${ }^{2}$ 1976, S. 131-157.

${ }^{24}$ Siehe zu diesem viel diskutierten Aspekt des Canto Michelangelo Picone, »Guittone, Guinizelli e Dante«, in: ders., Percorsi di lirica duecentesca. Dai Siciliani alla >Vita nova〈, Firenze, 2003, S. 169-184; Anthony Oldcorn, »Gone with the Wind: A Reading of Purgatorio XI«, in: Da Dante a Montale, a cura di Gian Mario Anselmi et alii, Bologna 2005, S. 35-63; Mario Marti, »Sui due Guidi di Purgatorio XI«, in: Da Dante a Montale, ebd., S. 65-75.

${ }^{25}$ Ich verstehe "gloria della lingua« sensu largo als Sprachruhm, während Köhler (wie Anm. 7), S. 219 die Semantik für meine Begriffe ein wenig zu stark verengt, indem er »lingua « ausschließlich auf die Sprache der Dichtung bezieht und übersetzt: »Ebenso hat auch in der Dichtersprache der eine Guido dem anderen Guido den Ruhm geraubt, [...].« 
Die Kritik ist sich weithin darin einig, dass diese anonyme Prophezeiung dem Dichter Dante und seinem künstlerischen Streben gilt. ${ }^{26}$ In der Argumentation Oderisis steht sie zunächst weniger im Zeichen der schmeichelhaften Vorhersage zukünftiger Größe an sich als im elliptischen Mahnen an deren kurze Dauer. Es wird auf diese Stelle noch einmal zurückzukommen sein.

Das in unserem Gesang avisierte positive Korrelat der superbia ist traditionsgemäß die humilitas. ${ }^{27}$ So heißt es bereits im Alten Testament: »Radices gentium superbarum arefecit Deus, / et plantavit humiles ex ipsis gentibus.« (Sir. 10, 18-19) Als edelste aller christlichen Tugenden steht die Demut dem Hochmut auch noch in der Commedia als Ziel aller Läuterung antithetisch gegenüber. Wo der Hochmut gewissermaßen die Degradation der menschlichen Natur anzeigt, indiziert die Demut deren vollkommene Erfüllung. Als höchster Grad an spiritueller Einkehr des Sühnenden stellt die umiltà damit gleichsam das Leitthema der gesamten zweiten Cantica dar. Auf dem Weg zu ihr wähnen sich alle auf dem Läuterungsberg Sühnenden. Repräsentiert wird das Streben nach Demut in Purgatorio XI in erster Linie von Oderisi. Den Moment der Umkehr hingegen versinnbildlicht in ihm kein anderer als Dante selbst, der in dem Miniaturmaler Oderisi ein alter ego erkennt, das ihm, dem überaus ehrgeizigen Dichter, den Spiegel vorhält. Oderisis Ermahnungen zeitigen ihre Wirkung darin, dass >Dante< bußfertig bekennt, seine Worte seien wahr und erfüllten sein Herz mit »rechter Demut« (Purg. XI, 119), so dass sein großer Stolz abschwelle. Unmittelbar anknüpfend an Oderisis in direkter Rede wiedergegebene $>$ Predigt $<$ heißt es: »E io a lui: «Tuo vero dir m'incora / bona umiltà, e gran tumor m'appiani« (Purg. XI, 118 f.) Das Ich bekennt sich damit ostentativ zum kognitiven Geschehen des Läuterungsweges, in dem die Einsicht in das eigene Fehlverhalten

\footnotetext{
${ }^{26}$ Bestätigt hat diese Auffassung zuletzt Pierantonio Frare, »Il nome, il volo, il numero. Lettura di >Purgatorio< XI«, in: Bellini, Eraldo / Girardi, Maria Teresa / Motta, Uberto (Hrsg.), Studi di letteratura italiana in onore di Claudio Scarpati, Milano 2010, S. 77-104, hier S. 84. Marini (wie Anm. 10), S. 82-86 schlägt hingegen vor, die Prophezeiung lediglich auf Dante als den stilnovistischen Dichter zu beziehen, während der Verfasser der Göttlichen Komödie wohl nicht gemeint sei.

${ }^{27}$ Siehe zur Demut in der Commedia konzise die Lemmata »umile« und »umiltà« von Francesco Tateo in der Enciclopedia dantesca, S. 818-819 u. S. 819821.
} 
die Möglichkeit einer wahrhaft tugendhaften conversio ad Deum eröffnet.

Aber auch körperlich zeugt das Ich von großer Demut, insofern als Dante sich in diesem Gesang als einen Hochmütigen inszeniert, der die Qualen der superbi mit dem Körper eines Lebendigen und daher umso stärker erleidet. Beschwert durch die »Last des Adamskörpers«, der ihn im Gegensatz zu den ihn umgebenden Seelen »umhüllt«, kommt Dante "gegen seinen Willen" am Berg nur langsam voran. (Purg. XI, 43 f.) Und er, der sich daran erinnert, »che tutto chin con loro andava" (Purg. XI, 78), empfindet die reuige Last, unter der er selbst voranschreitet, wie die von Stieren im Joch. So das prägnante Bild, mit dem der folgende Canto anhebt:

Di pari, come buoi che vanno a giogo, m'andava io con quell'anima carca, fin che $[\ldots]$ (Purg. XII, v. 1-3)

Geistig wie körperlich weist der Gesang Dante damit plastisch als einen im Prozess der Läuterung befindlichen superbo aus, dem das Bewusstsein für die Sündhaftigkeit des eigenen Ruhmstrebens deutlich auf den Leib geschrieben ist. Das Ich, so scheint es, löst damit das eingangs erwähnte Programm der doppelten purgatio mustergültig ein. Und der Hochmut, zumal in der Gestalt des künstlerischen Ehrgeizes, wird konkret als ein Problem in Szene gesetzt, das die Figur Dantes sowohl als Gegenstand der Darstellung als auch den Dichter im Kern betrifft. Es bestätigt sich damit das Urteil Chiavacci Leonardis, die den Sims der superbi als den Ort auf dem Läuterungsberg bezeichnet, den Dante als den ihm zugehörigen anerkenne und der ihn besser als alle anderen charakterisiere. $^{28}$

Doch beschränkt sich die Bedeutung der Tatsache, dass Dante sich in Purgatorio XI selbst als einen Hochmütigen inszeniert, der sich in Posen der humilitas anschickt, eine mutatio vitae zu vollziehen, keinesfalls

${ }^{28}$ In der »Introduzione« zu Purg. XI schreibt Chiavacci Leonardi (wie Anm. 15, S. 313): »Dei sette gironi della montagna, questo è quello che egli riconosce come suo, e suo è in realtà questo canto, dove la sua vicenda umana traspare nella trama del racconto [...].« Der Text selbst konfirmiert die zentrale Bedeutung des Hochmuts für die Ich-Figur bekanntlich in Purg. XIII, wenn Dante auf dem Sims der invidiosi gegenüber Sapia a posteriori die für ihn weiterhin größere Gefährdung durch das quälende Laster unseres Gesang eingesteht: »Troppa è piú la paura ond'è sospesa / l'anima mia del tormento di sotto, / che già lo 'ncarco di là giú mi pesa.« (Purg. XIII, 136-138) 
auf den Entwicklungsprozess des Protagonisten. Die einsetzende Demut nämlich, die der Figur hier zugeschrieben wird, verweist mittelbar darauf, dass das Ich den Weg durch die Hölle, auf den Läuterungsberg und in das Paradies in der Commedia nicht nur im eigenen Interesse unternimmt. Dante versinnbildlicht vielmehr allegorisch eine Mission, die ihn über sein individuelles Schicksal hinaus auch zum Gesandten eines kollektiven Erlösungsauftrags macht. Aufschlussreich ist es in diesem $\mathrm{Zu}-$ sammenhang, sich daran zu erinnern, dass der Protagonist seine Jenseitsreise von Beginn an im Namen eines übergeordneten Geschehens antritt. So bezieht sich bereits in Inferno I die Verirrung in dem »dunklen Wald «, die Begegnung mit den drei wilden Tieren und die auf diese Weise symbolhaft zum Ausdruck gebrachte Abkehr vom »rechten Wege«, welche Dante hier in Erinnerung ruft, nicht nur auf das Ich der Commedia. Genau besehen nämlich verweist die Ausgangslage Dantes allegorice zugleich auf den Zustand der Gesellschaft seiner Zeit. Deutlich macht diesen allegorischen Sinn der Pilgerfahrt Dantes die berühmte erste Prophezeiung, mit der Vergil dem um Hilfe Flehenden bei ihrer ersten Begegnung antwortet. Die Vision einer fernen Errettung von sündhafter Verstrickung durch den geheimnisvollen »veltro«, der dereinst die Bestie, welche das Ich bedroht, ${ }^{29}$ in die Hölle verbannen und auf italienischem Boden die ursprüngliche Herrschaft von »sapienza, amore e virtute« (Inf. I, 104) wiederherstellen wird, weitet der weise Dichter hier auf das Schicksal der »umile Italia« (Inf. I, 106 ff.) aus. ${ }^{30}$

${ }^{29}$ Ich folge in diesem Punkt der Argumentation von Giuglielmo Gorni, »Canto I«, in: Georges Güntert / Michelangelo Picone (Hrsg.), Lectura Dantis Turicensis, Bd. I, Firenze 2000, S. 27-38, der mit guten Gründen dafür plädiert hat, in den drei »fiere« in Analogie zur Dreifaltigkeit die Repräsentation des dreifaltigen Bösen zu sehen. Dafür spricht u.a., dass, nachdem Dante seine angsterfüllte Begegnung mit den Tieren geschildert hat, fortan stets nur noch von einer »bestia« die Rede ist.

${ }^{30}$ Die Passage lautet: " >A te convien tenere altro vïaggio<, / rispuose, poi che lagrimar mi vide, / >se vuo' campar d'esto loco selvaggio; / ché questa bestia, per la qual tu gride, / non lascia altrui passar per la sua via, / ma tanto lo 'mpedisce che l'uccide; / e ha natura sí malvagia e ria, / che mai non empie la bramosa voglia, / e dopo 'l pasto ha piú fame che pria. / Molti son li animali a cui s'ammoglia, / e piú saranno ancora, infin che 'l veltro / verrà, che la farà morir con doglia. / Questi non ciberà terra né peltro, / ma sapïenza, amore e virtute, / e sua nazion sarà tra feltro e feltro. / Di quella umile Italia fia salute / per cui morí la vergine Camilla, / Eurialo e Turno e Niso di ferute. / Questi la caccerà per ogne villa, / fin che l'avrà rimessa ne lo 'nferno, / là onde 'nvidia prima dipartilla.« (Inf., I, 91-111) 
Die Gleichsetzung von Dantes schuldhafter Verirrung mit der Geschichte des geschundenen Italien jedoch macht aus dem Fall des Protagonisten die Repräsentation eines kollektiven Zustands. Die selva oscura und die Anfechtungen durch die »bestia« bezeichnen somit per Analogie gleichermaßen die Situation des in Sünde verstrickten Ich wie den $\mathrm{Zu}-$ stand des ebenso sündigen zeitgenössischen Italiens. Letzteres zeichnet sich in den Augen des Autors, wie das Attribut »umile« anzeigt, dadurch aus, dass Italien die Demut, dass es die christliche Tugend der humilitas, die es eigentlich auszeichnen sollte, eingebüßt hat. Durch die Analogsetzung der Schuld des Einzelnen mit derjenigen der Gemeinschaft aber gewinnt Dantes Mission von Beginn an eine historische Dimension. Seine in Purgatorio XI evozierte Umkehr zur Demut dient nicht alleine der Erlösung seiner selbst, sondern darüber hinaus der des in Sünde gefallenen Italien. Die Tragweite des Gegensatzzusammenhangs von Hochmut und Demut reicht damit in der Commedia über die Figur des Protagonisten auf einen sensus historicus hinaus. Diese historische Dimension der Jenseitsreise jedoch, so hat Kablitz gezeigt, subsumiert Dante wiederum einer ontologischen Bedeutung, welche alles mundane Geschehen letztlich als Allegorie der Sündhaftigkeit alles Diesseitigen begreift. Als Ergebnis seiner Analyse der Sündenallegorie in Inferno I hält Kablitz fest: »Der sensus litteralis als ein sensus historicus bringt für Dante [...] die Wirklichkeit der Heilsgeschichte selbst ins Spiel: die Erfüllung der Zeit mit Gottes Gegenwart durch die Erlösungstat Christi. ${ }^{31}$

Der thematische Kern des enormen Bedeutungspotentials von Dantes allegorischer Ich-Figur aber liegt in besagtem Gegensatzzusammenhang von Hochmut und Demut. Die Bedeutung von Purgatorio XI besteht in diesem Kontext darin, dass der Gesang die ungemeine Spannung, die den beiden Polen des Läuterungsprozesses idealiter eignet, vielleicht wie kein zweiter in sich verdichtet und sie als solche nicht zuletzt in der Figur des personaggio-poeta ${ }^{32}$ ästhetisch erfahrbar macht. Soweit zum ersten Schritt dieser Lektüre, dem es darum gegangen ist, das moraltheologische Programm des Canto nachzuzeichnen.

\footnotetext{
${ }^{31}$ Andreas Kablitz, »Dantes poetisches Selbstverständnis (Convivio - Commedia)«, in: Winfried Wehle (Hrsg.), Die Schwierigkeiten (s)ich zu sagen, Frankfurt a.M. 2001, S. 17-57, Zitat S. 52.

${ }^{32}$ Die Rollen »Dantes« samt der mehrfachen Lesbarkeit, die sich daraus hinsichtlich der Geschichte des Ich ergeben, hat maßgeblich Gianfranco Contini erörtert in »Dante come personaggio-poeta della Commedia « $\left({ }^{1} 1958\right)$, zuletzt in: G. C., Un'idea di Dante, Turin 1976, S. 33-62.
} 
II.

Dies jedoch ist nur eine Seite der Medaille. Eine zweite Lektüre des Canto nämlich zeigt, dass der theologisch geschulten Ordnung in dem Gesang eine grundlegende Ambivalenz zu Eigen ist, welche die Gültigkeit der Doktrin erheblich relativiert. Die Ambivalenz besteht, dies sei vorweggenommen, hinsichtlich der menschlichen Vermögen, die in dem Canto als Urgrund der superbia angeprangert werden: Gemeint sind der Einsatz der menschlichen Geisteskraft sowie der Wille des Menschen hervorzuragen. Denn beide Qualitäten stehen bei allen Figuren, deren Läuterung der Gesang ins Bild setzt, keinesfalls ausschließlich als Äquivalent dafür, dass alle menschlichen Anstrengungen eitles Ruhmstreben bedeuten. Ganz im Gegenteil, unterschwellig attestiert der Canto dem Zusammenspiel des »ingegno« mit dem »disio de l'eccellenza« durchaus eine positive Funktion.

Konkret greifbar wird dies am Phänomen der »nominanza« (Purg. XI, 115), worunter die Namhaftigkeit einer Person zu verstehen ist, oder anders gesagt, ihre Berühmtheit. ${ }^{33}$ Hinter dem Begriff steckt semantisch die Tatsache, dass sich der Bekanntheitsgrad eines Menschen nicht zuletzt daran bemisst, wie häufig sein Name genannt wird. Sich einen Namen gemacht zu haben, bedeutet über einen langen Zeitraum allgemein namentlich bekannt zu sein. In dieser Bedeutung figurieren die Substantive »nome« (Purg. XI, 4, 60, 102), »voce« (Purg. XI, 103), der gelehrte »mondan romore« (Purg. XI, 100), aber auch sinnverwandte Syntagmen insgesamt nicht weniger als zehnmal in dem Gesang. ${ }^{34}$ Dies ist dort, wo das eitle Ruhmestreben angeprangert wird, nicht weiter verwunderlich, und so setzt Oderisi die Namensnennung schlichtweg mit einer selbstverliebten, hohlen Namenslust gleich: »Berühmtheit ist doch nur ein kurzes Lüftchen, das mal von hier kommt, mal von da und den Namen wechselt wie die Richtung « (Köhler S. 219). Als kardinales Symptom der superbia erscheint in unserem Canto damit eine concupiscentia nominum.

${ }^{33}$ Siehe zum Problemkomplex von curiositas und »nominanza« Teodolinda Barolini, The Undivine Comedy. Detheologizing Dante, Princeton 1992, 132142. Das Verhältnis von nome und superbia in Purg. XI jüngst Pierantonio Frare noch einmal grundlegend analysiert: »Il nome, il volo, il numero. Lettura di Purgatorio XI«, in: Bellini / Girardi / Motta, Studi di letteratura italiana (wie Anm. 26), S. 77-104, hier: 77-84.

${ }^{34}$ Siehe V. 95 f.: »[...] ora ha Giotto il grido / si che la fama di colui è scura«, V. 104: »[...] Toscana sonò tutta« (Kursivierungen M.S.). 
Wenn aber die Namensbegierde prominenter Ausdruck von Hochmut ist, bedeutet dies im Umkehrschluss, dass die angestrebte Demut mit Namenlosigkeit einherzugehen hat - ist doch, wie uns das Vater Unser eingangs belehrt, der einzige Name, den jedes Geschöpf loben soll, der »Name« des Herrn. ${ }^{35}$ Wahrhaft umile wäre demnach derjenige, dessen bußfertige Einkehr dahin gelangt ist, dass er der selbstbezüglichen Anerkennung durch die nominanza nicht mehr bedarf. Diesen unausgesprochenen Idealzustand aber repräsentiert im vorliegenden Gesang lediglich anfänglich der anonyme Chor der »ombre« (Purg. XI, 26), die sich betend von der »caligine del mondo« (Purg. XI, 30) reinigen. Die folgenden Begegnungen Dantes mit den drei Repräsentanten des Hochmuts hingegen zeichnen sich dadurch aus, dass alle an ihnen Beteiligten weit davon entfernt sind, sich unabhängig vom eigenen Namen, von der eigenen fama und damit jenseits der eigenen Lebensgeschichte $\mathrm{zu}$ begreifen. Ja, die Darstellungen des Adeligen Omberto Aldobrandeschi, des Miniaturmalers Oderisi da Gubbio sowie des Militärs Provenzan Salvani weisen die Namhaftigkeit jedes Einzelnen nicht etwa nur als unausweichliches Übel ihres irdischen Lebens oder als zu negierendes Durchgangsstadium auf dem Weg zu namenloser Demut aus. Sie können vielmehr genauso als Ausdruck eines durchaus stolzen Blicks zurück auf die eigene ruhmreiche Vergangenheit gelesen werden. Eine solche positive Selbstbezüglichkeit jedoch, die ihr identifikatorisches Potential aus der memoria an das eigene Leben schöpft, würde synekdochisch verstanden bedeuten, dass die auf der ersten cornice Buße tuenden Seelen, dem Hochmut, der sie zu Lebzeiten auszeichnete, noch immer weithin verhaftet sind. Diese Schlussfolgerung legt mittelbar Purgatorio X nahe, in dem mittels einer rhetorischen Exclamatio diejenigen als "hochmütige Christenmenschen « angeklagt werden, deren "geistiges Auge" erblindet ist, weil sie auf die »rückwärts gewandten Schritte«, sprich: die eigene Vergangenheit vertrauen, anstatt zu erkennen, dass wir Würmer sind, deren eigentliche Bestimmung es ist, »Engelsfalter zu werden, die unbehindert nach oben schweben sollen zum Gerechten!« (Köhler, S. 199)

O superbi cristian, miseri lassi,

che, de la vista de la mente infermi,

fidanza avete ne' retrosi passi,

non v'accorgete voi che noi siam vermi

35 »laudato sia 'l tuo nome e 'l tuo valore / da ogne creatura, com'è degno / di render grazie al tuo dolce vapore.« (Purg. XI, 4). 
nati a formar l'angelica farfalla, che vola a la giustizia sanza schermi? (Purg. X, 121-126)

Das Zitat ruft noch einmal die für den Läuterungsberg proklamierte Wandlung auf, welche von der sündhaften Verstrickung in Hochmut hin $\mathrm{zu}$ einer von aller Korruptheit befreiten engelsgleichen Demut führen soll. Wenn aber, wie im Folgenden zu zeigen sein wird, die demütig Buße Tuenden in Purgatorio XI sich noch immer in erheblichem Maße über ihre »ritrosi passi« definieren, führt dies zum Befund einer weit reichenden Ambivalenz. Dass Dante das Programm des Läuterungsberges in dem Gesang in solcher Weise verlebendigt, gilt es nun im Einzelnen darzulegen.

Nachdem Vergil der Schar der Betenden zur Erfüllung ihres Strebens »giustizia« und »pietà« (Purg. XI, 37) gewünscht und sie im Namen seines Schützlings nach dem kürzesten Weg gefragt hat, ${ }^{36}$ antwortet eine zunächst anonyme Stimme. Diese weist den beiden die Richtung, um die Sprache jedoch gleich darauf unvermittelt auf die Frage der Namhaftigkeit zu bringen. Dazu beklagt sie, dass sie ihr Gegenüber, das seinen Namen nicht nenne, obwohl es noch lebe (»cotesti, ch'ancor vive e non si noma«, V. 55), ${ }^{37}$ nicht ansehen kann. So könne sie nicht erkennen, ob

${ }^{36}$ Diesen an die Schattengestalten gerichteten Segenswünschen Vergils (Purg. XI, 37-39) geht die bedrückte Frage der Erzählstimme voraus, wie denn die Christenmenschen jenen Jenseitigen, die stets für sie beteten, in Wort und Tat helfen können: „Se di là sempre ben per noi si dice, / di qua che dire e far per lor si puote / da quei c'hanno al voler buona radice? « (Purg. XI, 31-33)

${ }^{37}$ Die Frage der »assenza del nome di Dante« (V. 84) an dieser Stelle ist meines Wissens erstmals Frare (vgl. Anm. 27) nachgegangen. Als Ergebnis seiner komplexen Argumentation deutet er die Nichtnennung als bewusste Verweigerung und als zeichenhaftes Symptom dafür, dass sich die Läuterung Dantes wie seine Hoffnung auf künftigen Ruhm in Purg. XI gewissermaßen nel mezzo del camin befinde: »Un silenzio che [...] credo che si possa interpretare come un riflesso della condizione di Dante, già salvo (e quindi proteso verso l'uomo perfetto, l'Adamo-Cristo), ma sempre sottoposto alla fatica del viaggio e alla tentazione del ritorno, che sarebbe una ricaduta verso il basso, verso l'uomo-bestia (l'Adamo caduto, l'Adamo-Lucifero); e ancora nonostante tutto vittima della superbia. Infatti, in questo episodio [...] il rifiuto di Dante di nominarsi si radica nell'attesa e nella speranza, da parte sua, di poter diventare tanto famoso da essere nominato da altri; attesa e speranza che verranno amaramente frustrate quando Dante sarà nominato, ma da Beatrice, e non per lodarne il nome, ma per rimproverarne aspramente il possessore. (S. 100) In demselben Sinne liest Frare die Tatsache, dass Dantes Name auch in der ersten Prophezeiung Oderisis (Purg. XI, 97-99) namentlich nicht genannt wird; vgl. ebd. S. 104. 
sie den anderen - gemeint ist Dante - kennt, und in ihm kein Mitleid für die eigenen Qualen erwecken. ${ }^{38}$ Damit aber ist der Bann gebrochen, und von einem unbändigen Drang getrieben gibt sich die Seele überaus stolz als Nachfahre aus dem toskanischen Hause Aldobrandeschi, der Grafen von Santafiora, zu erkennen. Dies tut sie, indem sie sich über eine ganze Terzine im herrschaftlichen Klang des Namens ihres alten Geschlechts ergeht und unverblümt die Bestätigung ihrer Berühmtheit von Dante einfordert:

Io fui latino e nato d'un gran Tosco:

Guiglielmo Aldobrandesco fu mio padre;

non so se 'l nome suo già mai fu vosco. (Purg. XI, 58-60)

Auch wenn sich die Figur samt ihrer Sippe im Folgenden wiederholt der »Arroganz« (Purg. XI, 62) und des »Hochmuts« (Purg. XI, 68) bezichtigt, wirkt dies angesichts der "glänzenden Taten«, derer sie das »antico sangue« (Purg. XI, 61) rühmt, und des überaus stolzen Duktus ihrer Worte wenig überzeugend. Der Leser gewinnt vielmehr den Eindruck, als wirke die »tiefe Verachtung«, die Omberto zu Lebzeiten »für jedermann « hatte (Purg. XI, 64), im Jenseits ungebrochen fort. ${ }^{39}$

Die Weise, in welcher der Läuterungsgang Oderisis charakterisiert wird, überspringe ich einstweilen bewusst, um zur Darstellung Provenzans, des dritten Hochmütigen zu kommen. Die Sequenz (Purg. XI, 120-142) unterscheidet sich von den vorangehenden darin, dass die Figur nicht selbst das Wort ergreift, sondern ihr Schicksal vom Miniaturmaler erzählt wird. Das Porträt, das er von Provenzan zeichnet, ist das eines machtgierigen (»presuntüoso«, Purg. XI, 122) Politikers, der dafür büßt, dass er »sich anmaßte, Siena ganz und gar unter seine Macht zu zwingen « (V. 122-123). Die Tat, die ihm gleichwohl die Aufnahme auf den Läuterungsberg bescherte, war, dass er sich, um das Lösegeld für einen von Karl von Anjou gefangen gehaltenen Freund aufzubringen, in der Zeit seiner größten Machtfülle aus freien Stücken auf den Zentralplatz in Siena setzte und, ich zitiere, »so verhielt, dass ihm - oder einem? - alle Pulse beb(t)en« - »si condusse a tremar per ogne vena."

\footnotetext{
${ }^{38}$ Im Wortlaut: »E s'io non fossi impedito dal sasso / che la cervice mia superba doma, / [...] guardere' io, per veder s'i' 'l conosco, / e per farlo pietoso a questa soma.« (Purg. XI, 52-57)

${ }^{39}$ Die zwei hier paraphrasierten Terzinen lauten: »L'antico sangue e l'opere leggiadre / d'i miei maggior mi fer sí arrogante, / che, non pensando a la comune madre, / ogn'uomo ebbi in despetto tanto avante, / ch'io ne mori', come i Sanesi sanno, / e sallo in Campagnatico ogne fante.« (Purg. XI, 61-66)
} 
(Purg. XI, 138). ${ }^{40}$ Die Kritik interpretiert diesen bewusst dunklen Vers ${ }^{41}$ im Allgemeinen dahingehend, dass Provenzan seine Mitbürger auf dem Platz gleichsam in einem Akt franziskanischer Demut um Almosen angebettelt und so in actu tiefe Reue an den Tag gelegt habe. ${ }^{42}$ Stierle jedoch hat in seiner Züricher Lectura des Canto dahingehend argumentiert, dass man diese von Dante nicht näher bestimmte Handlungsweise des »ruhmreichen« Sienesers - im Text ist anerkennend von seiner »opera" (Purg. XI, 142) die Rede ${ }^{43}$ - genauso gut als weiteren Akt hochmütiger Annmaßung lesen kann. ${ }^{44}$ Denn wer sich bar jeglicher Scham (»ogne vergogna disposta«, Purg. XI, 135) der öffentlichen Verachtung aussetzte, verleihe damit seiner eigenen Verachtung für den König wie für die Gemeinschaft demonstrativ Ausdruck. Auf diese Weise verwandle Provenzan die Selbsterniedrigung zugleich in ein Motiv des Triumphs. ${ }^{45}$ Damit aber trägt sein Hochmut, so Stierle, »dialekti-

${ }^{40}$ Die Infinitivkonstruktion lässt m.E. syntaktisch den Bezug offen, so dass sich das Beben aller Pulse nicht unbedingt auf die handelnde Figur des Provenzan beziehen muss. Die in dem Bild zum Ausdruck gebrachte compassio kann ebenso auf die Gegenwart der Rezipienten und damit Dantes Deutungsperspektive bezogen werden.

${ }^{41}$ Im nächsten Vers weist Oderisi seine Andeutungen explizit als `trobar clus aus: »Piú non dirò, e scuro so che parlo; « (Purg. XI, 139.

${ }^{42}$ Siehe in diesem Sinne stellvertretend den Stellenkommentar von Chiavacci Leonardi (wie Anm. 15), S. 344 f.

${ }^{43}$ Siehe vergleichend dazu die eindeutig positiv konnotierten »opere leggiadre« (Purg. XI, 61), derentwegen Omberto sein Geschlecht zuvor unumwunden rühmt.

${ }^{44}$ Karlheinz Stierle, »Canto XI«, in: Georges Güntert / Michelangelo Picone (Hrsg.), Lectura Dantis Turicensis, Bd. II: Purgatorio, Firenze 2001, S. 157172. In ähnlicher Weise gegen den Strich hat die Sequenz ansatzweise bereits früher Teodolinda Barolini (The Undivine Comedy, wie Anm. 33), S. 134-137, gelesen. Sie bezeichnet bereits als Charakteristikum des Canto »the dialectic between fame and evanescence $\ll$. (S. 137)

${ }^{45}$ Stierle, »Canto XI« (wie Anm. 44), S. 166 geht so weit, Provenzan verfolge mit der Selbstdemütigung eigentlich das Ziel, den eigenen Ruhm noch zu mehren: »[...] Provenzan umiliando se stesso accresce la propria gloria [...].« Eine Intention der Figur jedoch lässt sich m.E. aus der Passage kaum rekonstruieren, wie Frare, »Il nome, il volo, il numero« (wie Anm. 26), zurecht kritisiert: »un conto è rinonoscere nei personnaggi della prima cornice (Dante compreso) il permanere di tratti di superbia, altro è fare di loro (in particolare di Provenzan e di Dante) dei superbi che usano, più o meno astutamente, l'umiltà al fine di alimentare la loro vanità.« (S. 80) Dessen ungeachtet weist diese Sequenz jene re- 
sche« Züge, denn in seinem Verhalten koinzidieren umiltà und superbia gleichsam.

Diese meines Erachtens argumentativ plausible Lesart ${ }^{46}$ lässt aber auch die abschließende Prophezeiung, die Oderisi an Dante richtet, in einem ambivalenten Licht erscheinen. Der Canto endet damit, dass Oderisi seine opaken Worte mit dem Hinweis darauf abbricht, ihm, Dante, würden seine Florentiner Mitbürger bald so mitspielen, dass er sie dann selbst werde erklären können: »ma poco tempo andrà, che ' tuoi vicini / faranno sí che tu potrai chiosarlo." (Purg. XI, 140-141) Chiavacci Leonardi und andere lesen diese pathetische Vorhersage recht umstandslos als verschleierte Ankündigung des schmerzvollen Exils, das der historische Dante erleiden sollte. ${ }^{47}$ Vor der Folie der Doppeldeutigkeit von Provenzans vermeintlicher Demutsgeste freilich lässt sich die Prophezeiung bezüglich Dantes ebenso als inszenierte Vorwegnahme einer märtyrerhaften Apotheose des politischen Dante verstehen.

In den Beispielen der beiden ehemaligen Machtmenschen Ombertos und Provenzans manifestiert sich bis hierher eine grundlegende Ambivalenz, die darin besteht, dass Hochmut und Demut im Handeln der Figuren als kaum unterscheidbar inszeniert werden, dass sie strukturell gewissermaßen untrennbar zusammenfallen. Dies hat zur Folge, dass das moraltheologische Postulat eines Läuterungsprozesses, auf dem die humilitas die superbia ablöst, hier nicht überzeugend abgebildet wird. Es scheint im Gegenteil vielmehr so, als herrsche eine unausgesprochene Konkurrenz zwischen zwei Prinzipien, die im konkreten Fall keine deutliche Trennschärfe aufweisen. Diesen Befund bestätigt und erweitert die zentrale Episode des Gesangs, das lange Gespräch zwischen Dante und Oderisi (Purg. XI, 79-119), dem wir uns nun zuwenden wollen.

zeptive Ambivalenz auf, die darin besteht, dass sie mit guten Argumenten in zwei gegenläufige Richtungen gedeutet werden kann.

${ }^{46}$ Die jüngste italienische Forschung hingegen vertritt weiterhin die Auffassung, Provenzans Geschichte verkörpere »compiutamente nella Commedia la saldatura tomistica tra magnanimità e umiltà«. (Marini, »La gloria della lingua« (wie Anm. 10), S. 76) Siehe in diesem Sinne auch Frare, »Il nome, il volo, il numero« (wie Anm. 26).

${ }^{47}$ Chiavacci Leonardi (wie Anm. 15) deutet V. 140 f. wie folgt: »faranno in modo, esiliandoti dalla tua patria, che tu potrai ben commentare, cioè intendere il senso di quelle oscure parole; perché anche tu proverai, mendicando la vita (Par. VI 141), quello stesso fremito.« (S. 345) 
Nachdem ihn jener erkannt und zu sich gerufen hat, erkennt auch Dante den anderen und spricht ihn bewundernd mit den Worten an: »Oh, [...] bist du nicht Oderisi, / der Stolz von Gubbio und die Zierde der Kunst, / die man in Paris Illuminieren nennt? « (Köhler S. 215) Das italienische Original verwendet dabei in Vers 80 im Gegensatz zu Köhlers eleganterer Übertragung zweimal den Begriff der »Ehre«:

\author{
»Oh!«, diss' io lui, »non se' tu Oderisi, \\ l'onor d'Agobbio e l'onor di quell'arte \\ ch'alluminar chiamata è in Parisi? « \\ (Purg. XI, 79-81, Kursivierung M.S.)
}

Auf diese Ehrerbietung antwortet Oderisi, indem er zunächst, wie bereits erwähnt, demütig gesteht, inzwischen leuchteten die von einem anderen kolorierten Blätter heller, weshalb jenem jetzt alle Ehre zukomme, ihm hingegen nur noch zum Teil - »l'onore è tutto or suo, e mio in parte." (Purg. XI, 84) Die einschränkende Apposition aber verrät, dass auch derjenige, der in dem Gesang als das vorrangige Sprachrohr der christlichen Doktrin fungiert, dass auch Oderisi nicht ganz frei vom Laster der nominanza ist. Die schmeichelnde Anrede Dantes, so scheint es, hat ihn dazu verleitet, stolz einzuräumen, dass mit seinem Namen immerhin noch ein wenig Glanz verbunden bleibt. Entscheidender als diese Beobachtung, die zunächst lediglich unterstreicht, dass auch Oderisis purgatio nicht als vollendet gelten kann, ist die Frage, was genau Oderisi zu diesem Eingeständnis bewogen hat.

Bedeutsam scheint mir in dieser Hinsicht zu sein, dass Dantes Anrede den Begriff des onore verwendet und zwar mit anaphorischem Nachdruck gleich zweimal. Dies ist signifikant, weil der Text damit dem Künstler und alter ego Dantes ein Attribut zuordnet, das im zeitgenössischen Sprachgebrauch eindeutig für die uneingeschränkte Anerkennung höchster Tugend steht. ${ }^{48}$ Dies gilt auch für die damalige theologische Rede, der zufolge der appetitus honoris, das Verlangen nach Ehre, ebenso wie der appetitus gloriae an sich als moralisch neutraler Lohn der Tugendhaftigkeit angesehen wurde. Nur wenn das Ehrstreben unmäßig

${ }^{48}$ Die Tugendhaftigkeit des künstlerischen Ruhms realisiert sich dabei, wie Marini (»La gloria della lingua«, wie Anm. 10) betont, vor allem darin, dass das hervorragende Handeln im rechten Maß zwischen Hoffahrt und Feigheit erfolgt: »[...] un'>onrata impresa come quella della Commedia deve fuggire parimenti superbia e viltà ed essere animato da >buon adire $[. .$.$] per evitare di spingere il$ proprio ingegno artistico sulla scia del >folle volor senza ritorno di Ulisse.« (S. 71) Siehe in diesem Sinne ausdrücklich Inf. II, 121-132. 
ausfiel und auf ein falsches Ziel gerichtet war, wurde es thomistisch betrachtet zur Sünde; es galt dann als inordinatus appetitus honoris. ${ }^{49}$ Der Differenz dieser Begriffsprägungen entsprechend steht der Ruhm in dem Canto antithetisch den Sünden der superbia und der vana gloria gegenüber. Seiner ethischen Strahlkraft, so können wir folgern, ist es geschuldet, dass sich Oderisi zum durchaus selbstbewussten Geständnis seiner eingeschränkt anhaltenden Berühmtheit hinreißen lässt. Kaum zufällig greift er in Vers 84 eigens den Begriff auf, mit dem Dante ihm geschmeichelt hat: »l'onore è tutto or suo, e mio in parte."

Die Einführung der Kategorie des uneingeschränkt ehrvollen Ruhmes gibt zu zwei weiterführenden Überlegungen Anlass. Die erste betrifft das Fundament, auf dem die Ehre steht. Wenn sie, wie hier anzunehmen ist, thomistisch als praemium virtutis den Lohn für geleistete Tugend bezeichnet, dann folgt daraus, dass die Fähigkeiten, die der Einzelne zur Verwirklichung des Guten eingesetzt hat, ebenfalls tugendhaft sein müssen. Konkret heißt das im Falle Oderisis, dass der Ehrgeiz hervorzuragen, dessen er sich sühnend bezichtigt, zugleich den Ursprung jenes onore darstellt, der ihm aus Sicht Dantes unbestreitbar zukommt. Der »disio / de l'eccellenza ove mio core intese« (Purg. XI, 86 f.), erscheint auf dem ersten Rundsims des Läuterungsberges keinesfalls ausschließlich als hochmütig - er fungiert vielmehr im selben Moment als Garant einer »Kunst« (Purg. XI, 80), die ihrem Schöpfer im Urteil des auctor zu großer Ehre gereicht! Damit zeichnet sich ab, dass die Dantesche superbia eine Medaille mit zwei a priori gleichwertigen Seiten ist. Das Streben nach artistischer (und politischer) Vorherrschaft bezeichnet unter den Vorzeichen des christlichen Doktrin den Urgrund aller Sündhaftigkeit und erfordert daher eine bedingungslose purgatio. Im Zeichen des menschlichen Lebens hingegen benennt es ein Prinzip, welches den Antrieb allen ästhetischen und zivilisatorischen Fortschritts bildet und zugleich eine unbegrenzte Steigerung menschlicher Fähigkeiten bedingt. Es ist dieses anthropologische Konstituens aller Kulturgeschichte, das die von Oderisi angeführten drei Beispiele eitler artistischer Überbietung implizit zumindest auch repräsentieren. Bedenkt man

${ }^{49}$ Vgl. zu den Belegen erneut Patrick Boyde, Human vices (wie Anm. 19), S. 187 f. In der Quaest. Disp. de malo 9 nimmt Thomas eine dreifache Definition der vana gloria vor und charakterisiert sie dabei (drittens) als ein Ruhmstreben, das nicht auf das rechte Ziel hin - gemeint ist selbstredend die gloria Dei gerichtet ist: »Tertio modo dicitur gloria vana, quando gloria hominis non ordinatur ad debitum finem.« 
des Weiteren, dass das Argument des amor propriae excellentiae in der Rede Oderisis synekdochisch für alles »menschliche Können« überhaupt steht, ergibt sich daraus eine fundamentale Positivierung aller menschlichen Anstrengungen. Die Erfindungsgabe der Menschen, ihr Verstand, ihre Vermögen, alles »umane posse« (Purg. XI, 91) stehen demnach potentiell stets zugleich im Dienste sündigen Hochmuts wie tugendhafter Ehre. Der menschlichen Lebenszeit wird damit im Rahmen des Purgatorio de facto auch eine positive Funktion zuteil: Denn der sub specie aeternitatis eitle Drang zur Überbietung des anderen begründet implizit zugleich den Grundsatz politischer, kultureller, künstlerischästhetischer Fortschritte des Einzelnen wie einer Gemeinschaft in der Geschichte. Die vor allem bei Provenzan zu beobachtende Koinzidenz von Hochmut und Demut ist demnach kein singulärer Einzelfall, sondern Symptom einer grundlegenden Ambivalenz, die Dantes Behandlung der superbia kennzeichnet. Diese Ambivalenz ist freilich keinesfalls mit einer etwaigen intentio auctoris zu verwechseln; sie besteht vielmehr auf hermeneutischer Ebene in Hinblick auf die je doppelte Lesbarkeit des Texts.

Die zweite, weiterführende Überlegung hinsichtlich der Ehre betrifft deren zeitliche Gültigkeit. Wie geschildert, besteht Oderisis wesentliches Argument gegen die vana gloria in deren Kurzlebigkeit, ja seine Kritik des Hochmuts reduziert sich bemerkenswerterweise beinahe gänzlich auf den Aspekt ihrer eklatanten zeitlichen Begrenztheit. $\mathrm{Zu}$ überzeitlicher oder gar ewiger Berühmtheit, so besagt das moraltheologische Argument, können die Menschen aufgrund ihrer irdischen Verdienste per definitionem niemals gelangen. Doch gibt es in unserem Text eine Stelle, die auch der Relativierung dieses Glaubenssatzes den Weg bahnt. Es handelt sich mit Vers 93 erneut um einen vermeintlich beiläufigen Nachsatz. Er schließt an das vorhin zitierte Postulat an, der eitle Ruhm des menschlichen Könnens währe doch nur so kurz wie das Grün auf den Wipfeln, und lautet: »se non è giunta da l'etati grosse!« (Purg. XI, 93) - »es sei denn, es folg(t)en barbarische Zeiten!« überträgt Köhler (S. 217) ins Deutsche. Diese vermeintlich beiläufige Einschränkung mindert den exemplarischen Status der von Oderisi evozierten Maler und Dichter, deren große Namen im nächsten Moment schon wieder durch neue verblasst seien, erheblich. Denn der in sämtlichen mir zugängigen Editionen mit einem Ausrufezeichen versehene emphatische Nachsatz besagt grundsätzlich nichts anderes, als dass es Zeitumstände geben kann, unter denen der Ruhm einer Person sehr wohl von Dauer ist. Stellt Dante seine Gegenwart mit der Dekadenz der »umile Italia«, 
die ihre Demut eingebüßt habe, nicht just als eine solche Zeit dar? Die Frage, wie lange Ehre und Ruhm bestehen bleiben, wird damit vom Sprachrohr des Läuterungsprogramms grundsätzlich den kontingenten Wechselfällen der irdischen Zeit unterstellt. ${ }^{50}$

Nun wird man einwenden, dass die Relativierung des Ubi-suntMotivs, die Oderisi hier anklingen lässt, nur im Rahmen der menschlichen, innerweltlichen Zeit gilt. Das Jenseits und der Kern der christlichen Doktrin bleiben davon unangetastet, so scheint es. Doch auch dies ist nur vordergründig der Fall, wie die bereits angesprochene Prophezeiung indiziert, mit der Oderisi Dante voraussagt, er werde die bisherigen Heroen der "gloria della lingua e einst aus dem Nest vertreiben. Die unmittelbare Intention dieser warnenden Worte Oderisis ist es, sein Gegenüber zur Demut anzuhalten, da sie ihm zu verstehen geben, auch er werde die Vorherrschaft als Dichter zweifellos bald anderen überlassen müssen. Zieht man hingegen als Subtext dieser Prophezeiung die oben skizzierte Mission Dantes als Dichter der Commedia hinzu, drängt sich eine gegenläufige Lesart auf: Indem sich Dante, wie gesehen, insgeheim als denjenigen inszeniert, der die eigene Jenseitsreise nicht nur im Sinne des eigenen Heils, sondern zur Erlösung Italiens, ja der Menschheit insgesamt von ihrer Sünde unternimmt, steht der Bericht seiner Jenseitsreise im Zeichen der göttlichen Wahrheit. Die Vorstellung aber, das Ich handle im Auftrag des Allmächtigen, impliziert, dass dem Verfasser der Commedia, sofern er sein Werk vollendet, ewiger Ruhm verheißen ist. Um im Bilde Oderisis zu bleiben: Dante wird sich aus dem Nest nicht vertreiben lassen, da er (der Anlage seines Weltgedichts folgend) auserwählt ist, jene göttliche Wahrheit zu verkünden, die jenseits aller menschlichen Zeitlichkeit und innerweltlicher Kontingenz besteht. Die irdische Zeit kann seinen poema sacrato (Par. XXIII, 62) demnach gerade nicht ein- und überholen. So gelesen, tadelt Oderisis erste Prophezeiung Dantes vana gloria nicht bloß und hält ihn zur conversio ad Deum an, sie sagt ihm im selben Zug auf dem Weg, den er beschreitet, insgeheim die Erlangung der vera gloria voraus. Dies aber heißt nichts anderes, als dass Oderisis verklausulierter Aufruf zur Um-

\footnotetext{
${ }^{50}$ Marianne Shapiro schreibt zu dem Prinzip historischer Kontingenz, dem der Ruhm damit letztlich unterstellt wird, treffend: »Not lasting maturity but rather cyclical rebirth and destruction attend the affairs of individuals as of their states and aggregates. Time plays all kinds of tricks of hierarchical reversal, eclipsing the spurious durability of fame. « Marianne Shapiro, »Homo artifex: A Rereading of Purgatory XI«, in: Tibor Wlassics (Hrsg.), Lectura Dantis, Charlottesville 1993, S. 59-69, Zitat S. 65.
} 
kehr auch im Sinne einer zukünftigen Apotheose des Dichters der Commedia gedeutet werden kann. Die Prophezeiung hat in diesem Lichte die Funktion einer poetologischen mise en abyme. Argumentiert man streng im Rahmen des moraltheologischen Programms des Canto muss eine solche Lesart freilich als blasphemische Hybris erscheinen.

Gestützt wird die hier vorgeschlagene Deutung indirekt von einem Canto, in dessen Zentrum ebenfalls das Schicksal ehrenvoller Dichter steht. Die Rede ist vom vierten Höllengesang, dem der edlen Heiden, die im Limbus weilen, weil ihnen der Aufstieg in das Reich Christi $a$ priori nicht vergönnt ist. Hier findet sich die berühmte Szene, in der Dante auf die vier großen Dichter der lateinischen Antike, Homer, Horaz, Ovid und Lukan trifft. Apostrophiert werden auch sie mit dem Attribut des onore, und zwar in der Weise, dass Dante den Begriff in einem kunstvollen Polyptoton in nur neun Versen rhetorisch eindrucksvoll fünf Mal variiert. ${ }^{51}$ Inhaltlich aussagekräftig für meine Argumentation ist diese intratextuelle Korrespondenz insofern, als Vergil Dante in dieser Passage ausdrücklich darüber belehrt, der ehrenvolle Ruf der heidnischen Dichter, der auf Erden noch nachklinge, verschaffe ihnen auch im Himmel Gnade und mehre sich somit weiter:

E quelli a me: »L'onrata nominanza

che di lor suona sú ne la tua vita,

grazia acquista in ciel che sí li avanza.«(Inf. IV, 76-78)

Für die heidnischen Dichter, die immerhin auf ewig in die Hölle verdammt sind, sieht die Autorität des directeur de conscience Vergil demnach vor, dass ihre mundane Berühmtheit gleichwohl die göttliche Gna-

\footnotetext{
${ }^{51}[\ldots]$ orrevol gente possedea quel loco. / »O tu ch'onori scïenzïa e arte, / questi chi son c'hanno cotanta onranza, / che dal modo de li altri li diparte?«. / E quelli a me: »L'onrata nominanza / che di lor suona sú ne la tua vita, / grazïa acquista in ciel che sí li avanza«. / Intanto voce fu per me udita: / »Onorate l'altissimo poeta; / l'ombra sua torna, ch'era dispartita.« (Inf. IV, 72-81, Kursivierungen M.S.)

${ }^{52}$ Dies ist übrigens neben Purg. XI, 115 die einzige Stelle in der Commedia, an der Dante den Begriff der nominanza verwendet. Köhler (wie Anm. 7) versteht das zweite Hemistichion von V. 78 philologisch korrekt im Sinne einer allgemeinen Aufwertung und übersetzt: »Ihr ehrenvoller Ruf, der in deinem Leben oben noch nachklingt, bewirkt auch im Himmel für sie Gnade, und so hebt er sie hervor.« (S. 63, Kursivierung M.S.) Meine freiere Paraphrase hingegen, die dem Originalzitat vorangestellt ist, bezieht das avanzamento bewusst auf die »onrata nominanza« als dessen Ursprung.
} 
de bewirken kann. Der Gebrauch des Verbs »acquistar« vermittelt dabei auch im Altitalienischen prominent den Verdienstgedanken, der jenes Verhältnis von Ursache und Wirkung im menschlichen Handeln beinhaltet, welches dem Gedanken der grazia Dei fremd ist. Ist aber eine Erlösung, die auf dem »ehrenvollen Ruf« zu Lebzeiten beruht, für die freilich edlen, aber doch paganen Dichter der Antike denkbar, wie viel offener muss diese dann jenen stehen, die wie die Protagonisten des elften Gesangs, Dante eingeschlossen, sich im Läuterungsprozess befinden $?^{53}$

Vor dieser Folie wirkt die dreifache Zuschreibung des onore auf Oderisi in unserem Gesang wie ein entferntes Echo des Polyptotons zu Ehren der Alten in Inferno IV. In der Rede Vergils wird die unerhörte Aufwertung der Dichtung durch das historische Beispiel der heidnischen poetae seiner Zeit exemplifiziert. Oderisis offensichtlich an Dante gerichtete Prophezeiung überbietet diese eklatante Infragestellung der christlichen Gnadenlehre noch insofern, als sie diese Möglichkeit in der Person desjenigen aktualisiert, der die Göttliche Komödie schreibt.

Im Hinblick auf unsere übergeordnete Fragestellung aber besagt dieser Befund, dass die Ambivalenz, welche der Darstellung der superbia innewohnt, ihren wesentlichen Ausdruck nicht zuletzt in der Figur des Dante-poeta findet, der in sich die gegenläufigen Konzepte von christlicher Demut und dichterischem Ruhmstreben vereint. ${ }^{54}$ Das Medium sei-

${ }^{53}$ Hollander, »Dante's Self Laureation« (wie Anm. 22) geht hingegen davon aus, dass die Auffassungen des Heiden Vergil nicht unbedingt vom christlichen Autor der Commedia geteilt werden und urteilt daher hinsichtlich Inf. IV, 76-78: »the high estimate of fama is distinctly Virgilian and pagan, reflecting exactly what is also wrong in Brunetto's view of how Dante may be saved (Inferno XV, 55-60), where what counts is the public success of one's work, where the sport one sails for is fame, not salvation.« (S. 38) Stichhaltige Indizien dafür, dass Dante die Lehre seines maestro in diesem Punkt negiert, liefert Hollander freilich nicht.

${ }^{54}$ Einen solchen »intimo dissidio« bestätigt Marini, »La gloria della lingua« (wie Anm. 10), wenn er schreibt, es sei »indubbio che l'idea dell'arte legata alla vanità della mondana snominanzar (S. 115) che ne consegue rappresenti un tassello difficilmente armonizzabile con quanto Dante dice altrove in materia e con lo stesso sistema poetico su cui poggia l'edificio della Commedia.« (S. 79) Im Gegensatz zu der hier vertretenen Auffassung jedoch geht es Marini im Folgenden um den Nachweis, dass hier lediglich eine »apparente contraddizione« vorliege, die sich auflöse, wenn man die »evoluzione interna al pensiero del poeta « (ebd.) in Betracht ziehe. Das hier aufgeworfene Problem nämlich erübrige sich angesichts des göttlichen Erlösungsauftrags, in dessen Zeichen die Commedia 
ner Verkündigung ist die eigene Dichtung. In sie aber hat Dante all seinen ingegno investiert, sie ist Ausdruck eines ausgeprägten disio de l'eccellenza. Damit, so lässt sich schließen, müssen die menschlichen Anlagen, die der Canto für den Hochmut auf Erden verantwortlich macht, genauso als unerlässliche Voraussetzung einer gotteswürdigen Kunst aus Menschenhand gelten. Die genannten Fähigkeiten erweisen sich in dieser Perspektive in ihrer Wertigkeit a priori als weder sündhaft noch tugendhaft, sondern als Teil einer anthropologischen Ausstattung, welche die Menschen gleichermaßen zum Guten wie zum Schlechten einsetzen können. ${ }^{55}$ In dieser Erkenntnis scheint mir ein wesentlicher Teil des Aussagepotentials von Purgatorio XI zu liegen.

III.

Über dies hinaus hat die nähere Betrachtung der vorgeblichen Beispiele reuiger Abkehr vom Hochmut zur Demut gezeigt, dass sich alle Figuren in ihrem Auftreten zumindest partiell auch durch das Gegenteil von gottesfürchtiger humilitas auszeichnen. Auf diese Weise stellen sie in actu den Läuterungsprozess infrage, dessen Exemplifizierung sie gleichzeitig für sich in Anspruch nehmen. Begreift man nun die purgatio gemäß des Bußsakraments als Dreischritt, bedeutet dies, dass das Läuterungsgeschehen in dem Gesang höchst unvollständig dargestellt ist: Überzeu-

sich, wie gesehen, selbst begreift: »il poema di Dante non può essere sottomesso alla legge di Oderisi. In quanto veicolo di un messaggio profetico ispirato direttamente dalla Grazia [...], la Commedia si pone al di là del suo raggio d'azione strettamente circoscritto alla contigenza dell'arte terrena [...].«(S. 85)

${ }^{55}$ Von zentraler Bedeutung für die Entscheidungen, die diese Wahlmöglichkeit grundsätzlich impliziert, ist in der Commedia die Kategorie des liberum arbitrium. Als Indiz dafür, welchen Rang dieses im Läuterungsprozess zumal des Ich hat, sei hier lediglich auf die Abschiedsworte verwiesen, die Vergil auf dem Gipfel des Purgatorio an Dante richtet. Im irdischen Paradies, das Dante gezielt spiegelbildlich als Gegenort zur »selva selvaggia aspra e forte« von Inferno I als »divina foresta spessa e viva« (Purg. XXVIII, 2) angelegt hat, bescheinigt der antike Dichter dem nun vollends von seinen Sünden gereinigten Dante die volle Wiedererlangung des freien Willens, dem er fortan in seinem Handeln einzig verantwortlich sei: »Tratto t'ho qui con ingegno e con arte; / lo tuo piacere omai prendi per duce; [...] Non aspettar mio dir piú né mio cenno; / libero, dritto e sano è tuo arbitrio, / e fallo fora non fare a suo senno; / per ch'io te sovra te corono e mitrio.« (Purg. XXVII, 130-131, 139-142; Kursivierung M.S.) 
gend bringt er lediglich die contritio cordis zur Anschauung, was sich darin manifestiert, dass die Hochmütigen und der mitleidende Dante die ihnen auferlegten körperlichen Qualen reuig annehmen. Das Sündenbekenntnis hingegen, die confessio oris, fällt zumindest im Falle von Omberto und Provenzan zweideutig aus. Und auch das Bekenntnis Dantes, die Worte Oderisis erfüllten ihn mit gottesgläubiger »Demut« und ließen seinen »großen Stolz abschwellen« (Purg. XI, 119), büßt durch seine im selben Atemzug vorgebrachte, investigative Frage an Konsistenz ein. Die Terzine lautet:

E io a lui: »Tuo vero dir m'incora bona umiltà, e gran tumor m'appiani; ma chi è quei di cui tu parlavi ora?« (Purg. XI, 118-120)

Das imperative Drängen der mit dem konzessiven »ma« eingeleiteten Aufforderung an Oderisi, ihm sogleich den Namen und das Schicksal Provenzans zu enthüllen, lenkt die Aufmerksamkeit auf die Frage als das semantische Zentrum der Terzine. Die beiden vorangehenden Verse hingegen wirken in dem syntaktischen Gefüge gewissermaßen inhaltlich nachgeordnet, so dass bezweifelt werden darf, ob die Rede des anderen Dantes Herz wirklich mit »bona umiltà« erfüllt (»m'incora«, Purg. XI, 118). Es hat vielmehr den Anschein, als gerate das Demutsbekenntnis angesichts der unbändigen curiositas Dantes zu einem Lippenbekenntnis. Vor allem aber die satisfactio operis, welche sich in den guten Taten des Büßenden manifestiert, bleibt in dem Canto eine Leerstelle. Denn das Handeln der Figuren hat in Wort und Tat in auffälliger Weise beinahe ausschließlich die Frage der eigenen Namhaftigkeit oder jene Dritter zum Thema. Es bleibt selbstbezüglich, während etwa selbst eine ansatzweise Reflexion über Wiederherstellung der eigenen Einsichtsfähigkeit in das summum bonum gänzlich ausbleibt. Damit aber findet der intellektuelle Prozess der Läuterung schlichtweg nicht statt, ebenso wie die sich Läuternden mit Ausnahme Oderisis der persönlichen Vergangenheit verhaftet bleiben.

Was aber, so muss man fragen, spricht dann im elften Gesang überhaupt für die Umkehr der superbi zur Demut? Göttliche Anzeichen dafür sind in dem Gesang nicht zu erkennen, was sich auch daran zeigt, dass die Anbetungen des Vater Unser in der Darstellung der drei Toskaner kein Echo finden, was wiederum darauf schließen ließe, dass sie in irgendeiner Form von Gott auserwählt wären. Und nach einem konsistenten >formalen « Kriterium, das für alle gleichermaßen als Voraussetzung des Aufstiegs auf den Läuterungsberg gilt, wird man ebenfalls ver- 
geblich suchen. Dies gilt zumal für die Vorstellung eines reuigen Schuldbekenntnisses zu Lebzeiten, mittels dessen sich der Mensch des purgatorium als würdig erweisen kann. Oderisi führt diesen kausalen Nexus zwischen der reuigen Konfession, die er vollzog, »als ich noch sündigen konnte«, und seiner Aufnahme in die zweite Cantica explizit zu seinen Gunsten ins Feld, wenn er eingesteht: »e ancor non sarei qui, se non fosse / che, possendo peccar, mi volsi a Dio. « (Purg. XI, 89-90) Ebenfalls ausdrücklich im Dienste dieses Gedankens steht seine Erzählung davon, wie der machtgierige Provenzan eines Tages auf dem Marktplatz von Siena öffentlich für einen Freund bettelte (Purg. XI, 133-138). Denn sie antwortet unmittelbar auf einen Einwand Dantes, der verwundert gefragt hat, wie es denn sein könne, dass jenem der Zutritt hier oben so schnell gewährt worden sei, wo doch jene Seelen, die erst im Angesicht des Todes Buße getan hätten, gewöhnlich >ein zweites Leben lang im Antipurgatorio ausharren müssten. ${ }^{56}$ Der dritte im Bunde, Umberto Aldobrandeschi, hingegen dementiert das Argument demonstrativ. Er erklärt unumwunden, er habe die seinen Hochmut angemessenen Lasten nicht »tra 'vivi« geschleppt und müsse dies daher nun eben »hier unter den Toten« tun:

E qui convien ch'io questo peso porti per lei [la superbia, M.S.], tanto che a Dio si sodisfaccia, poi ch'io nol fe' tra' vivi, qui tra ' morti. (Purg. XI, 70-72)

Das Bild der Lasten, die Umberto als Buße für seine Sünden zu Lebzeiten nicht schulterte, impliziert zweifellos auch das Ausbleiben eines Sündenbekenntnisses, welches der satisfactio operis ja vorausgehen müsste. Damit wird klar, dass dieser Hochmütige auf den Läuterungsberg steigen durfte, obwohl er das pönitentiale Kriterium, das im Weiteren für die beiden anderen superbi veranschlagt wird, nicht erfüllt. Eine Vergleichbarkeit ist also in diesem Punkt nicht verlässlich gegeben, ja Dantes Selektionsmaßstab für die Aufnahme in den Bewährungsraum des Purgatorio erweist sich an dieser Stelle als ausgesprochen inkohärent. Das denkbare Gegenargument, die fehlende Bußfertigkeit des lebendigen Umberto stelle kein Problem dar, weil die Abfolge der drei Toskaner in der Logik des Gesangs eine Steigerung hin zu einem Mehr

\footnotetext{
${ }^{56} \gg \mathrm{E}$ io: ^Se quello spirito ch'attende, / pria che si penta, l'orlo de la vita, / qua giú dimora e qua sú non ascende, / se buona orazïon lui non aita, / prima che passi tempo quanto visse / comme fu la venuta lui largita?« (Purg. XI, 127132).
} 
an gottgefälliger Demut beschreibe, scheint mir nicht schlagkräftig zu sein. Denn die Frage nach den Voraussetzungen für die Aufnahme in das Purgatorio (ebenso wie in das Paradiso) ist kategorialer Art und in jedem einzelnen Fall neu zu prüfen. Mit dem Hinweis auf die moralische Entwicklung, welche die Syntagmatik der Commedia auch innerhalb der Canti zweifelsohne zum Ausdruck bringt, lässt sich dieser Punkt kaum verrechnen.

Wenn demnach das diesseitige Sündenbekenntnis nicht als valides Kriterium für die "venuta" (Purg. XI, 132) der drei Toskaner auf den Berg der Läuterung fungiert, bleibt zu fragen, woran sich ihre Aufnahme in die zweite Cantica denn dann bemisst. Es scheint, als sei einzig ausschlaggebend dafür paradoxerweise die nominanza, die ihnen allererst der auctor der Commedia verschafft! Dieses Kriterium lässt sich dahingehend zuspitzen, dass es letzten Endes Ruhm und Ehre sind, die ausgewählte sündige Seelen in der spezifischen Weise adeln, dass Dante ihnen die Aufnahme in das Who is who seiner Zeit beschert. ${ }^{57}$ Zur Bedingung für die Aufnahme Umbertos, Oderisis und Provenzans in das Purgatorio wird damit, dass der Verfasser der Commedia ihnen ebenso wie sich selbst Fähigkeiten zuschreibt, die herausragend sind und so die außerordentliche Namhaftigkeit eines jedes einzelnen von ihnen begründen. Damit kehrt sich die nach mittelalterlichem Verständnis realistische Überzeugung, dass die Namen das Ergebnis der Dinge sind (nomina sunt consquentia rerum) gewissermaßen um und macht insgeheim dem Grundsatz res sunt consequentia nominum Platz. ${ }^{58}$ Dies aber bedeutet nichts anderes, als dass die Erlösung der Seelen in dem Gesang in geradezu radikaler Weise vom Menschen her gedacht wird. Denn seine narrative Logik beruht, genau besehen, auf einer allzu menschlichen Ethik des öffentlichen Erfolgs. Dies erklärt auch, wieso unser Canto ge-

${ }^{57}$ Barolini, The Undivine Comedy (wie Anm. 33), hat bereits ähnlich argumentiert, indem sie schreibt: »[...] the text, being historically grounded, requires names, cannot help but celebrate them.« (S. 135)

${ }^{58}$ Der berühmte »pensamento« der Vita Nova lautet: »lo nome d'Amore è si dolce a udire, che / impossibile mi pare che la sua propria operazione sia ne le più / cose altro che dolce, con ciò sia che li nomi seguitino le nominate cose, sì come è scritto: >Nomina sunt consequentia rerum.« (Dante Alighieri, Vita no$v a$, a cura di Guglielmo Gorni, Torino 1996, 6, 4, S. 65 f.). Den Gedanken, dass Purg. XI diesen Grundsatz gleichsam auf den Kopf stellt, hat Oldcorn (»Gone with the wind«, wie Anm. 24, S. 108) pointiert formuliert und für Oderisi dahingehend zugespitzt, »that the miniaturist owes his enduring fame not to anything the historical Oderisi actually did but exclusivley to his appearance in Dante's poem [...]« (S. 109). 
radezu gezwungen ist, das »umane posse« entgegen der Doktrin, die er selbst predigt, signifikant aufzuwerten: Der Versuch, eine Vermittlung zwischen nichtiger Welt und verlässlicher Gottesordnung aus den kontingenten Bedingungen der irdischen Zeit und des diesseitigen Lebens herzuleiten, kann notwendigerweise nicht von den produktiven Vermögen der Menschen absehen. Es ist daher nur konsequent, wenn die selbstsüchtige Nutzung jener Fähigkeiten nicht nur als Wurzel aller Sünde gebrandmarkt wird, sondern de facto ebenso als die Quelle einer Kunst erscheint, die in ihrem Streben nach ästhetischer Vollkommenheit die Annäherung an das Göttliche sucht.

Das close reading des elften Purgatoriumsgesangs mündet, so lässt sich bilanzieren, in den Befund einer latenten Konkurrenz zweier Wege zu Gott. ${ }^{59}$ Während das räsonierte Vater Unser am Beginn des Canto noch einmal den »direkten Weg«, die »via diritta $($ Inf. I, 3) in sein Reich proklamiert, weisen den Menschen die drei Begegnungen Dantes insgeheim einen »altro vïaggio « $(\operatorname{Inf}$. I, 91). Er besteht im lebenspraktischen Weg einer via terrena. Auf dieser darf man nicht, wie auf dem ersten, betend auf die grazia Dei hoffen, sondern muss alle eigenen Fähigkeiten zur Mehrung von Ruhm und Ehre nutzen, in der Hoffnung, so Zutritt zu Dantes Läuterungsberg zu erlangen. Diese irdische Herleitung der moraltheologischen Jenseitslehre führt in dem Gesang zur Überlagerung zweier konträrer Bestimmungen der natura hominis - einerseits der theologisch-systematischen, die den Menschen sub specie aeternitatis entwirft, und andererseits der historisch-kontigenten, welche sich an den Bedingungen des Lebens der Menschen bemisst.

Die latente Konkurrenz, die sich aus dieser Konstruktion ergibt, lässt Dantes Projekt einer harmonischen Verbindung zwischen sündigem Diesseits und seligem Jenseits als äußerst prekär erscheinen. Bemerkenswert daran ist zum einen, dass Purgatorio XI dieses unerhörte Problem erst eigens namhaft macht und es im selben Zug kaschiert. Zum anderen vollzieht Dante auf diese Weise unter der Hand eine unerhörte Aufwertung der menschlichen Vermögen. Sie erscheinen in dem Canto nicht nur negativ, als Urgrund der miseria hominis, sondern ansatzweise und verschleiert ebenso als Ursprung der dignitas hominis.

Derjenige, der diese anthropologische Grundspannung wenige Jahrzehnte nach Dante aufgriff und erstmals zum dominanten Gegenstand

${ }^{59}$ Siehe zu der bereits im Convivio ausgeprägten Wege-Metaphorik Dantes grundlegend Teodolinda Barolini, »Purgatory as Paradigm: Traveling the New and Never-Before-Traveled Path of this Life/Poem« in: dies., The Undivine Comedy (wie Anm. 33), S. 99-121. 
der poetischen Reflexion erhob, war Francesco Petrarca. Dafür steht sein frühneuzeitlicher Entwurf eines unhintergehbar zerrissenen Ichs, das sich im Secretum und in den Rerum vulgarium fragmenta am Problem einer unmöglichen conversio animi abarbeitet. In Dantes Commedia ist dieser Konflikt erst im Ansatz erkennbar und noch nicht offen thematisch. Doch deutet er sich deutlich erkennbar in der grundlegenden Ambivalenz an, welche die Behandlung des Hochmuts in Purgatorio XI kennzeichnet. Wie bewusst dem Verfasser diese Ambivalenz gewesen sein mag, das sei dahingestellt. Für den Autor dieser Lectura Dantis jedoch erweist sich die doppelte Lesbarkeit im Ergebnis nicht nur als Signum dieses Canto, sondern der Danteschen Heilsanthropologie überhaupt. 
